\title{
Growing Random Sequences
}

\author{
I. Krasikov, G.J. Rodgers and C.E. Tripp \\ Department of Mathematical Sciences, \\ Brunel University, Uxbridge, Middlesex UB8 3PH, U.K.
}

\begin{abstract}
We consider the random sequence $x_{n}=x_{n-1}+\gamma x_{q}$, with $\gamma>0$, where $q=0,1, \ldots, n-1$ is chosen randomly from a probability distribution $P_{n}(q)$. When all $q$ are chosen with equal probability, i.e. $P_{n}(q)=1 / n$, we obtain an exact solution for the mean $\left\langle x_{n}>\right.$ and the divergence of the second moment $\left\langle x_{n}^{2}>\right.$ as functions of $n$ and $\gamma$. For $\gamma=1$ we examine the divergence of the mean value of $x_{n}$, as a function of $n$, for the random sequences generated by power-law and exponential $P_{n}(q)$ and for the non-random sequence $P_{n}(q)=\delta_{q, \beta(n-1)}$.

PACS numbers: 02.50.cw, 05.40.-a, 89.75Hc.
\end{abstract}

Keywords: Random sequences 


\section{INTRODUCTION}

Random sequences form a fundamental part of many models in fields ranging from science and technology to sociology and economics. From the random walk, models of packet transport on a network to models of income distribution and share price movement, a random sequence plays some role in the basic model.

Recently there has been much interest in the behaviour of the random Fibonacci series

$$
F_{n+1}=F_{n} \pm \alpha F_{n-1},
$$

where the plus or minus sign is taken with equal probability. It has been shown $[1,2]$ that there is a critical value of $\alpha, \alpha_{c} \approx 0.703$, such that, as $n \rightarrow \infty,\left|F_{n}\right|$ diverges exponentially when $\alpha>\alpha_{c}$ and decays exponentially to 0 for $\alpha<\alpha_{c}$. Problems of this type are technically very similar to a variety of problems in one-dimensional disordered systems [3], such as the Anderson model of electrons in a metal with impurities. In this paper we consider the random sequence

$$
x_{n}=x_{n-1}+\gamma x_{q}
$$

with, $\gamma>0$ and without loss of generality, $x_{0}=1$, where $q$ is a random variable chosen from the integers $q=0,1, \ldots, n-1$ with probability $P_{n}(q)$. This is a generalisation of the sequence produced by $P_{n}(q)=1 / n$ and $\gamma=1$, which was recently considered in [4]. There it was shown that asymptotically $<x_{n}>\sim n^{-1 / 4} \exp (2 \sqrt{n})$ as $n \rightarrow \infty$ and that the system exhibits multiscaling so that the typical behaviour of the sequence will deviate substantially from the behaviour of the average [4].

In general the mean of the sequence in Eq.(2) exhibits at least two different types of behaviour. Heuristically speaking, if $P_{n}(q)$ is such that it is dominated by $q$ of order $n$, then $<x_{q}>$ will be of order $x_{n-1}$ and the sequence will grow exponentially. Conversely if $P_{n}(q)$ is dominated by $q$ of order 1 , then $x_{q}$ will be of order $x_{0}$ and $\left\langle x_{n}>\right.$ will grow linearly in $n$. Between these two extremes other, more complex, types of behaviour are possible. In this paper we consider the mean and variance of the sequence generated by a uniform $P_{n}(q)$, as well as the sequences generated with a power-law and exponential $P_{n}(q)$, and the non-random sequence $P_{n}(q)=\delta_{q, \beta(n-1)}$. In the latter cases we find the critical values of the parameters in these distributions that determine the onset of linear and exponential growth, as well as determining the behaviour of the sequence between these regions. 


\section{RANDOM SEQUENCES}

In this and the next two sections we consider random sequences in which the random variable $q$ is chosen from a distribution $P_{n}(q)$ which is seperable. In other words $P_{n}(q)=$ $P(q) / b_{n}$ where $P(q)$ is independent of $n$ and

$$
b_{n}=\sum_{q=0}^{n-1} P(q) .
$$

In sequences of this type the average value of $x_{n}, A_{n}=<x_{n}>$, obeys

$$
A_{n}=A_{n-1}+\frac{\gamma}{b_{n}} \sum_{q=0}^{n-1} P(q) A_{q} .
$$

For general $P(q)$, when $\gamma=1$, multiplying this equation through by $b_{n}$, and subtracting the equivalent expression for $n+1$, reveals

$$
A_{n+1}-2 A_{n}+\frac{b_{n}}{b_{n+1}} A_{n-1}=0 .
$$

There is an interesting connection between Eq.(5) and orthogonal polynomials. It is well known that the recurrence

$$
f_{k+1}(x)-x f_{k}(x)+a_{k} f_{k-1}(x)=0 \quad a_{k}>0, f_{-1}=0, f_{0}=1,
$$

defines a family of symmetric monic polynomials with respect to a positive measure on the real line [5]. Thus, $A_{n}$ in Eq.(5) may be viewed as the value of the corresponding

orthogonal polynomial $f_{k}(x)$ with $a_{k}=\frac{b_{k}}{b_{k+1}}$ at $x=2$. Moreover, as $f_{k}(2) \neq 0$, the measure will be supported on a subset of $[-2,2]$. A linear change of variables yields the polnomials $q_{k}(x)=2^{-k} f_{k}(2 x)$ orthogonal on a subset of $[-1,1]$, satisfying

$$
q_{k+1}(x)-x q_{k}(x)+\frac{a_{k}}{4} q_{k-1}(x)=0 .
$$

Thus if we consider the sequence $P(q)=1$ and hence $b_{n}=n$, then we obtain the equation for $A_{n}$ in [4],

$$
A_{n+1}-2 A_{n}+\frac{n}{n+1} A_{n-1}=0 .
$$

The solution of this equation is in general $A_{n}=2^{n} P_{n}^{\lambda}\left(1 ; \frac{1}{2}, 0\right)$, where $\lambda$ is either $\frac{1}{2}$ or $\frac{3}{2}$ and $P_{n}^{\lambda}(x ; a, b)$ is a Pollaczek polynomial [5]. This is equal to the $\mathrm{n}^{\text {th }}$ Laguerre polynomial [6], $L_{n}(-1)$. Thus the exact solution for the average of $x_{n}$ is

$$
<x_{n}>=L_{n}(-1)
$$


As $n \rightarrow \infty$ we recover

$$
<x_{n}>\sim k_{1} n^{-1 / 4} \exp (2 \sqrt{n})
$$

with $k_{1}=1 / 2 \sqrt{e \pi} \approx 0.1711$ [7]. This asymptotic form was obtained in [4] by using the WKB method [8] on Eq.(8).

Using the same approach, we can easily show that for $\gamma \neq 1$ but $P(q)=1$ and hence $b_{n}=n$, for large $\mathrm{n}$,

$$
A_{n+1}-\left(2+\frac{\gamma-1}{n+1}\right) A_{n}+\frac{n}{n+1} A_{n-1}=0
$$

which has an exact solution $<x_{n}>=L_{n}(-\gamma)$. Asymptotically, $<x_{n}>\sim k_{\gamma} n^{-1 / 4} \exp (2 \sqrt{\gamma n})$.

In [4], it was shown numerically that when $P(q)=1$ and $\gamma=1$ the average of the sequence does not characterise its growth, and that the $\mathrm{k}^{\text {th }}$ moment grows faster than the $\mathrm{r}^{\text {th }}$ moment for all $r<k$. We can see this analytically by calculating the asymptotic growth of the $2^{\text {nd }}$ moment of the sequence with $P(q)=1$ and general $\gamma>0$. This can be done by introducing two averages

$$
V_{n}=<x_{n}^{2}>\quad \text { and } \quad M_{n}=\sum_{r=0}^{n-1}<x_{n} x_{r}>.
$$

Using Eq.(2) it is a simple matter to show that $V_{n}$ and $M_{n}$ obey the coupled iterations

$$
(n+1) V_{n+1}-\left(2 n+(\gamma+1)^{2}\right) V_{n}+(n+2 \gamma) V_{n-1}=2 \gamma\left(M_{n}-M_{n-1}\right)
$$

and

$$
(n+1) M_{n+1}-(2 n+2 \gamma+1) M_{n}+n M_{n-1}=(n+\gamma+1) V_{n}-n V_{n-1} .
$$

These equations can be written in the continuum limit, and then obey the coupled second order differential equations

$$
(t V(t))^{\prime \prime}-(2 \gamma+1) V^{\prime}(t)-\gamma^{2} V(t)=2 \gamma M^{\prime}(t)
$$

and

$$
(t M(t))^{\prime \prime}-M^{\prime}(t)-2 \gamma M(t)=(t V(t))^{\prime}+V^{\prime}(t)+\gamma V(t)
$$

In the limit $t \rightarrow \infty$, we can assume that $V(t) \sim t^{\phi} \exp (\delta \sqrt{t})$ and $M(t) \sim t^{\phi+1 / 2} \exp (\delta \sqrt{t})$. Substituting these forms into Eqs. $(15,16)$ and equating the leading order terms gives

$$
\delta=\sqrt{2 \gamma\left(4+\gamma+\sqrt{16+\gamma^{2}}\right)}
$$


When $\gamma=1$ we have $\delta=\sqrt{2(5+\sqrt{17})} \approx 4.27$. This compares well with [4], where the value $\delta \approx 4.3$ was obtained numerically. Note that, dropping pre-factors, the mean diverges as $\exp (2 \sqrt{\gamma n})$ and the second moment as $\exp (\delta \sqrt{n})$, so that as $\delta>2 \sqrt{\gamma}$ for all $\gamma,<x_{n}^{2}>$ diverges faster than $\left\langle x_{n}>\right.$ for all $\gamma$. As $\gamma \rightarrow \infty, \delta \rightarrow 2 \sqrt{\gamma}$ and as $\gamma \rightarrow 0$ then $\delta \rightarrow 4 \sqrt{\gamma}$.

\section{POWER-LAW $P_{n}(q)$}

Taking

$$
P(q)=(q+1)^{\alpha}
$$

and $\gamma=1$ yields four different classes of behaviour for $\alpha>-1, \alpha=-1,-2<\alpha<-1$ and $\alpha<-2$. We will deal with these in turn.

\section{A. $\alpha>-1$}

Here we have $b_{n} / b_{n+1}=1-(\alpha+1) / n+O\left(1 / n^{2}\right)$ for large $n$ and hence Eq.(5) becomes

$$
A_{n+1}-2 A_{n}+A_{n-1}=\frac{\alpha+1}{n} A_{n-1} .
$$

As before, this equation can be solved exactly, this time in terms of generalised Laguerre polnomials [6]. In particular, $A_{n} \sim L_{n}^{(-\alpha)}(-(\alpha+1))$. Hence as $n \rightarrow \infty$ we have

$$
A_{n} \sim c_{\alpha} \frac{1}{n^{\frac{2 \alpha+1}{4}}} \exp \{2 \sqrt{(\alpha+1) n}\}
$$

with $c_{\alpha}=(\alpha+1)^{(2 \alpha-1) / 4} e^{-(\alpha+1) / 2} / 2 \sqrt{\pi}$.

B. $\alpha=-1$

When $\alpha=-1$ we have $b_{n} / b_{n+1}=1-1 /(n+1) \log n+O\left(1 / n(\log n)^{2}\right)$ and

$$
A_{n+1}-2 A_{n}+A_{n-1}=\frac{1}{(n+1) \log n} A_{n-1}
$$

for large $\mathrm{n}$. Using the WKB [8] approximation we find that

$$
A_{n} \sim \frac{1}{\sqrt{n} \log n} \exp \left\{2 \sqrt{\frac{n}{\log n}}\right\} .
$$


C. $-2<\alpha<-1$

When $\alpha<-1$ we have $b_{n} / b_{n+1}=1-(n+1)^{\alpha} / \zeta(-\alpha)+O\left(n^{\alpha-1}\right)$ where $\zeta$ is the Riemann zeta function [9]. Hence for large $n$ Eq.(5) can be rewritten as

$$
A_{n+1}-2 A_{n}+A_{n-1}=\frac{(n+1)^{\alpha}}{\zeta(-\alpha)} A_{n-1}
$$

and using the WKB approximation [8] yields

$$
A_{n} \sim \frac{1}{n^{\frac{\alpha}{4}}} \exp \left\{\frac{2}{\alpha+2} \frac{n^{1+\alpha / 2}}{\sqrt{\zeta(-\alpha)}}\right\} .
$$

for $-2<\alpha<-1$. For $\alpha=-2$ the divergent asymptotic behaviour of $A_{n}$ is purely power-law with

$$
A_{n} \sim n^{\frac{1}{2}+\frac{1}{\sqrt{\zeta(2)}}}
$$

where the exponent $1 / 2+1 / \sqrt{\zeta(2)} \approx 1.108$ is greater than 1 .

\section{D. $\alpha<-2$}

When $\alpha<-2$ then the right hand side of Eq.(23) can be neglected and as $n \rightarrow \infty$, $A_{n} \sim n$.

\section{EXPONENTIAL $P_{n}(q)$}

Here we consider $P(q)=a^{q}$. When $a=1$ the solution in Eq.(9) is recovered. When $a<1$ then $b_{n} \rightarrow 1 /(1-a)$ as $n \rightarrow \infty$ and $A_{n}=n$. When $a>1$ then $b_{n} \sim a^{n} /(a-1)$ as $n \rightarrow \infty$ and hence

$$
A_{n} \sim\left[1+\sqrt{1-\frac{1}{a}}\right]^{n}
$$

\section{NON-RANDOM SEQUENCE}

Consider the non-random sequence

$$
x_{n}=x_{n-1}+x_{\beta(n-1)}
$$


where $0 \leq \beta \leq 1$ is fixed. If $\beta=0$ then $x_{n}=n$ whereas if $\beta=1$ then $x_{n}=2^{n}$. For $0<\beta<1$ we can solve the asymptotics of this sequence by converting Eq.(27) to a continuous firstorder non-local differential equation

$$
\frac{d x}{d t}=x(\beta t)
$$

where $t$ is the continuous counterpart of $n$. By substituting a power series solution for $x(t)$ into Eq.(28) and solving for the coefficients, we can find

$$
x(t)=\sum_{r=0}^{\infty} \frac{t^{r} \beta^{\frac{1}{2} r(r-1)}}{r !} .
$$

For large $t$ this summation is dominated by the term $r \sim \log (t) / \log (1 / \beta)$ and hence it is possible to evaluate the summation for large $t$ and show that for large $n$

$$
x_{n} \sim \exp \left\{\frac{(\log n)^{2}}{2 \log \left(\frac{1}{\beta}\right)}\right\} .
$$

\section{SUMMARY}

We have generalised previous studies to model random sequnces with a tunable memory. We have obtained an exact solution for the mean of the sequence $x_{n}=x_{n-1}+\gamma x_{q}$ when $q=$ $0,1,2, \ldots, n-1$ is chosen at random with probability $P_{n}(q)=1 / n$. We showed analytically how the $2^{\text {nd }}$ moment $\left\langle x_{n}^{2}>\right.$ diverges faster than the mean $\left.<x_{n}\right\rangle$. We also considered more general forms of $P_{n}(q)$, power-law, exponential and the non-random sequence $P_{n}(q)=$ $\delta_{q, \beta(n-1)}$. We found that these sequences exhibit exponential growth when $P_{n}(q)$ is dominated by $q \sim n$ and linear growth when $P_{n}(q)$ is dominated by $q \sim 1$. Between these two extremes an intermediate type of growth occurs. We were able to calculate this growth and determine the boundaries of the different types of behaviour, which are summarised in Table 1. Though the results in these sections were obtained for $\gamma=1$, the critical values of the parameters at the boundaries of the different regimes are valid for all $\gamma>0$, a general $\gamma$ merely changes the form of the divergence in the intermediate regime.

This sequence, although much simpler than the random Fibonacci sequence studied in $[1,2]$, as no negative numbers are allowed, displays a surprising rich phase space and a wide range of different types of behaviour. 


\section{ACKNOWLEDGEMENTS}

We would like to thank D. F. Scrimshaw for useful discussions. 
[1] D. Viswanath, Math. Comput. 691131 (2000).

[2] M. Embree and L.N. Trefethen, P. Roy. Soc Lond. A Mat. 4552471 (1999).

[3] J. M. Luck, Systémes Désordonnés Unidimensionnels, (Aléa-Saclay, France) 1992.

[4] E. Ben-Naim and P.L Krapivsky, J. Phys. A 35 L557 (2002).

[5] T. S. Chihara, An Introduction to Orthogonal Polynomials, (Gordon and Breach, London) 1978.

[6] M. Abramowitz and I.A. Stegun, Handbook of Mathematical Functions, (Dover, New York) 1975.

[7] G. Szego, Orthogonal Polynomials, (AMS, Rhode Island) 1939.

[8] E. J. Hinch, Perturbation Methods, (CUP, Cambridge) 1991.

[9] I.S. Gradshteyn and I. M. Ryzhik, Table of Integrals, Series and Products, (Academic Press, London) 1980. 
TABLE I: Summary of Results

\begin{tabular}{|c|c|c|c|}
\hline & Linear & Intermediate & Exponential \\
\hline$P_{n}(q) \sim(q+1)^{\alpha}$ & $\alpha<-2$ & $\alpha>-2$ & - \\
\hline$P_{n}(q) \sim a^{q}$ & $a<1$ & $a=1$ & $a>1$ \\
\hline$P_{n}(q)=\delta_{q, \beta(n-1)}$ & $\beta=0$ & $0<\beta<1$ & $\beta=1$ \\
\hline
\end{tabular}

